\title{
Le référentiel règlementaire
}

\author{
O. COUASNON ${ }^{1, \mathrm{a}}$, P. ROINE ${ }^{2}$, F. FOURNET $^{3}$, G. ABÉLA $^{4}$, E. MARTIN $^{5}$
}

\begin{abstract}
RÉSUMÉ La radiographie industrielle est soumise à la règlementation «générale " (radioprotection, prévention des risques, coordination, détention et utilisation de matériel contenant des sources radioactives, etc.) ainsi qu'à un certain nombre de règles spécifiques (formation au Certificat d'Aptitude à Manipuler les Appareils de Radioscopie Industrielle et de Radiographie Industrielle - CAMARI, conception des gammagraphes, conditions d'utilisation, etc.). Quelques informations sur des normes relatives à la conception des appareils de gammagraphie et au balisage sont également rappelées. Enfin, il est souligné que le travail réalisé n'a pas pour vocation une recherche d'exhaustivité.
\end{abstract}

ABSTRACT Regulatory guide.

Industrial radiography is subjected to the "general" regulation (protection against radiation, risk prevention, coordination, detention and use of material containing radioactive sources, etc. Like with a certain number of specific rules (training with the Certificate of Aptitude To handle the Industrial Radiography and Fluoroscopes CAMARI, design of the gammagraphes, conditions of use, etc.). Some information on standards relating to the design of the gammagraphy apparatuses and the beaconing is also pointed out. Lastly, it is stressed that work carried out does not have the role a search for exhaustiveness.

Keywords: Industrial radiography / gammagraphy / legislation / standardization / radiation protection / zoning / theft / accident

Pour ce premier chapitre, il nous est apparu nécessaire de faire un rappel de la règlementation encadrant la radiographie industrielle. L'utilisation des appareils de radiographie industrielle utilisant le rayonnement gamma (activité de gammagraphie industrielle) représente certainement l'activité à plus fort enjeu en termes de sécurité des contrôles radiographiques (notamment lors de chantiers « extérieurs »). L'atelier $1 \mathrm{~s}$ 'est donc focalisé sur la gammagraphie industrielle pour établir le référentiel réglementaire de radioprotection correspondant.

Nous aborderons tout à tour la règlementation générale, la règlementation spécifique à la radiographie industrielle, et l'aspect normatif. Il est souligné que le travail réalisé n'a pas pour vocation une recherche d'exhaustivité.

Animateur

Institut de radioprotection et de sûreté nucléaire, Direction de la radioprotection de l'Homme, Service d'études et d'expertise en radioprotection, Unité d'expertise en radioprotection des travailleurs et de la population, IRSN/DRPH/SER/ UETP, BP 17, 92262 Fontenay-aux-Roses Cedex, France.

2 CEA INSTN/AECO, Antenne de Cherbourg Octeville, ZA Les VINCITS, 143 chemin de la Crespinière, 50130 Cherbourg Octeville, France.

3 Autorité de sûreté nucléaire, Division d'Orléans, 6 rue Charles de Coulomb, 45077 Orléans Cedex 2, France.

4 EDF, Division ingénierie nucléaire, 1 place Pleyel, 93282 Saint-Denis Cedex, France.

5 COFREND, 1 rue Gaston Boissier, 75724 Paris Cedex 15, France. 


\section{Référentiel réglementaire - liste des textes applicables}

\subsection{Réglementation "générale"}

La règlementation générale relative à la radioprotection repose essentiellement sur les Codes du travail et de la santé publique. Ces Codes sont des recueils organisés de la plupart des textes législatifs et règlementaires applicables en matière de droit du domaine concerné.

Certains textes transposant les directives européennes en droit national, modifient le Code de la santé publique et le Code du travail en introduisant des dispositions en matière de protection contre les rayonnements ionisants, notamment :

- pour le Code du travail : articles L.230-1 et suivants; articles R.231-73 et suivants (analyse des postes de travail, démarche d'optimisation de la radioprotection, zonage radiologique, classement et suivi des travailleurs, formations des travailleurs, etc.) ;

- pour le Code de la santé publique : articles L.1333-1 et suivants (limites d'exposition du public, déclarations et autorisations préalables, suivi des sources, etc.).

Il faut préciser que des travaux de recodification du Code du travail, lancés le 15 février 2005 par le ministre chargé du travail, ont abouti :

- le 13 mars 2007, à la publication par ordonnance de la partie législative du nouveau Code du travail (annexe de l'ordonnance n²007-329 du 12 mars 2007 relative au Code du travail, partie législative - JO, 13 mars 2007, p. 4740). Cette partie législative du nouveau Code du travail doit entrer en vigueur le $1^{\text {er }}$ mai 2008, tel que prévu par l'article 2.X de la loi no2008-67 du 21 janvier 2008 (JO, 22 janv. 2008, p. 1122) ratifiant l'ordonnance n`2007-329 du 12 mars 2007 relative au Code du travail (partie législative) ;

- le 12 mars 2008, à la publication par décret de la partie règlementaire du Code du travail, annexée sous forme de publication spéciale au Journal officiel (annexe du décret n ${ }^{\circ} 2008-244$ du 7 mars 2008 relatif au Code du travail, partie règlementaire-JO, 12 mars 2008, p. 4482). Le décret n²008-244 vient préciser que la partie règlementaire du nouveau Code du travail doit entrer également en vigueur le $1^{\mathrm{er}}$ mai 2008 (art. 11). Cette nouvelle partie règlementaire est composée d'articles identifiés soit par un « $\mathrm{R} »$, correspondant aux dispositions relevant d'un décret en Conseil d'État, soit par un « $\mathrm{D}$ », pour les dispositions relevant d'un décret simple (art. 1).

Ce nouveau Code du travail recodifié « à droit constant » (sans modification du fond du droit même si des ajustements sont cependant effectués : la notion d'employeur 
vient se substituer à celle du chef d'établissement par exemple) doit entrer en vigueur le $1^{\mathrm{er}}$ mai 2008. Les références au Code du travail utilisées par la suite sont celles en vigueur jusqu'au $1^{\mathrm{er}}$ mai 2008 , ceci compte tenu de la date de rédaction, mais les concordances entre ancien et nouveau code sont disponibles à l'adresse suivante : http://www.travail-solidarite.gouv.fr ; on peut également souligner le travail important de mise à disposition des informations relatives à ces modifications règlementaires sur le site de l'Association pour les techniques et les sciences de la radioprotection (ATSR) : http://www.atsr-ri.asso.cc-pays-de-gex.fr/.

Outre ces digressions relatives aux codes, il faut rappeler que la règlementation générale repose donc sur les principaux textes suivants Disponibles sur le site http://www.legifrance.gouv.fr :

- Ordonnance 2001-270 du 28 mars 2001. Elle a traduit dans le Code de santé publique les trois grands principes (issus de consensus internationaux) fondamentaux de la radioprotection :

- aucune activité conduisant à un risque d'exposition aux effets des rayonnements ionisants ne peut être entreprise si elle n'est pas justifiée par un apport supérieur au détriment qu'elle est susceptible d'occasionner ;

- dès lors, il doit être recherché une optimisation de cette activité pour réduire les expositions aux rayonnements ionisants au niveau le plus bas qu'il est raisonnablement possible d'atteindre ;

- en tout état de cause, des limites d'exposition aux rayonnements ionisants sont fixées, et elles ne pourront pas être dépassées.

- Décret n²002-460 du 4 avril 2002 relatif à la protection générale des personnes contre les dangers des rayonnements ionisants, au régime général des autorisations et déclarations, à l'acquisition, la distribution, l'importation, l'exportation, la cession, la reprise et l'élimination des sources radioactives ainsi qu'aux contrôles.

- Décret $n^{\circ} 2007-1582$ du 7 novembre 2007 relatif à la protection générale des personnes contre les dangers des rayonnements ionisants et modifiant le Code de la santé publique. Les modifications introduites concernent notamment la réalisation des mesures de radioactivité et les régimes d'autorisation et de déclaration de certaines activités nucléaires. Il achève la transposition de la directive 2003/122/Euratom du conseil de l'Union européenne du 22 décembre 2003 relative au contrôle des sources radioactives scellées de haute activité et des sources orphelines, et prend en compte les nouvelles prérogatives accordées à l'Autorité de sûreté nucléaire (ASN), introduites par la loi n²006-686 du 13 juin 2006 relative à la transparence et à la sécurité en matière nucléaire.

- Décret n²003-296 du 31 mars 2003 relatif à la protection des travailleurs contre les dangers des rayonnements ionisants. La législation française en matière de radioprotection des travailleurs a fait l'objet d'une refonte 
importante en 2003 par ce décret qui visait à compléter la transposition de la directive 96/29/Euratom du conseil de l'Union européenne du 13 mai 1996 fixant les normes de base relatives à la protection sanitaire de la population et des travailleurs contre les dangers résultant des rayonnements ionisants. Les dispositions ont été insérées dans le Code du travail au livre II, titre III, chapitre Ier, section VIII (partie règlementaire, décrets pris en conseil d'état).

- Décret n²007-1570 du 5 novembre 2007 modifiant les dispositions du Code du travail relatives à la protection des travailleurs contre les dangers des rayonnements ionisants. Les modifications concernent notamment les contrôles techniques des dosimètres, les modalités de réalisation des contrôles techniques d'ambiance, la formation des travailleurs utilisant des appareils de radiologie industrielle, la formation et les missions de la personne compétente en radioprotection.

- Décret n92-158 du 20 février 1992 complétant le Code du travail et fixant les prescriptions particulières d'hygiène et de sécurité applicables aux travaux effectués dans un établissement par une entreprise extérieure.

- Décret $n^{\circ} 2006-1454$ du 24 novembre 2006 modifiant la nomenclature des installations classées.

- Arrêté du 8 octobre 1990 fixant la liste des travaux pour lesquels il ne peut être fait appel aux salariés sous contrat de travail à durée déterminée ou aux salariés des entreprises de travail temporaire modifié par l'arrêté du 12 mai 1998 (interdiction d'accès pour les travailleurs à contrat à durée déterminé et pour les intérimaires si le débit de dose est susceptible d'être supérieur à $2 \mathrm{mSv} / \mathrm{h}$ ).

- Articles L.122-3-17 et L.124-22 du Code du travail ; prescriptions relatives respectivement aux salariés sous contrat à durée déterminée ou sous contrat de travail temporaire (« prorata temporis »).

- Arrêté du 26 octobre 2005 définissant les modalités de contrôle de radioprotection en application des articles R.231-84 du Code du travail et R.1333-44 du Code de la santé publique.

- Arrêté du 15 mai 2006 relatif aux conditions de délimitation et de signalisation des zones surveillées et contrôlées et des zones spécialement réglementées ou interdites (« arrêté zonage »).

- Arrêté du 26 octobre 2005 relatif aux modalités de formation de la personne compétente en radioprotection et de certification du formateur.

- Arrêté du 21 décembre 2007 modifiant l'arrêté du 26 octobre 2005 relatif aux modalités de formation de la personne compétente en radioprotection et de certification du formateur (JO, 28 décembre 2007).

- Arrêté du 30 décembre 2004 relatif à la carte individuelle de suivi médical et aux informations individuelles de dosimétrie des travailleurs exposés aux rayonnements ionisants. 


\subsection{Règlementation spécifique}

La règlementation spécifique repose sur les textes règlementaires relatifs à la conception et aux conditions d'utilisation du gammagraphe sont listés ci-dessous :

- Décret n ${ }^{\circ 5-968}$ du 27 août 1985 définissant les conditions d'hygiène et de sécurité auxquelles doivent satisfaire les appareils de radiologie industrielle utilisant le rayonnement gamma (conception d'un gammagraphe).

- Arrêté du 11 octobre 1985 fixant le contenu et les règles d'utilisation des documents de suivi nécessaires à l'application des dispositions de l'article $22 \mathrm{du}$ décret nº5-968 du 27 août 1985 (maintenance périodique et carnet de suivi).

- Arrêté du 25 juin 1987 relatif au certificat d'aptitude à manipuler les appareils de radioscopie industrielle et de radiographie industrielle (CAMARI).

- Arrêté du 2 mars 2004 fixant les conditions particulières d'emploi applicables aux dispositifs destinés à la radiographie industrielle utilisant le rayonnement gamma. Cet arrêté a pour but de renforcer la règlementation de cette activité afin d'éviter que l'appareil ne soit utilisé par des personnes non qualifiées (formation, assistant, prévention du vol) et de prévenir une surexposition involontaire de l'opérateur (contrôle de la position de la source, signalisation de la position de la source sur l'appareil, contrôle systématique du débit de dose à l'aide d'un radiamètre).

- Arrêté du 21 décembre 2007 (publié au JO du 28 décembre 2007) définissant les modalités de formation et de délivrance du certificat d'aptitude à manipuler les appareils de radiologie industrielle (CAMARI). Cet arrêté prévoit son entrée en vigueur dans un délai de six mois après sa publication au JO, soit au 28 juin 2008. À cette date, il annulera et remplacera définitivement l'arrêté du 25 juin 1987 modifié qui avait fixé les précédentes conditions d'obtention du CAMARI.

- Arrêté du 21 décembre 2007 (publié au JO du 28 décembre 2007) portant homologation de la décision n²007-DC-0074 de l'Autorité de sûreté nucléaire du 29 novembre 2007 fixant la liste des appareils ou catégories d'appareils pour lesquels la manipulation requiert le certificat d'aptitude mentionné au premier alinéa de l'article R.231-91 du Code du travail. En effet, La manipulation d'un appareil de gammagraphie équipé de sources radioactives ou d'une installation de radiologie industrielle dotée d'un générateur électrique (à poste fixe ou mobile) demeure soumise à l'obtention du CAMARI. Aux appareils déjà soumis au CAMARI ont été rajoutés sur cette liste les accélérateurs de particules utilisés à des fins industrielles et certains équipements radiologiques de contrôles de fret dans les ports et aéroports. L'article R.231-91 du Code du travail, introduit par le décret $\mathrm{n}^{\circ} 2007-1570$ du 5 novembre 2007, maintient toujours le principe d'un certificat d'aptitude pour manipuler des appareils de radiologie industrielle figurant sur une liste établie par l'Autorité de sûreté nucléaire (ASN), mais ce certificat est désormais délivré par l'IRSN. 


\subsection{Normes}

Il convient de citer l'aspect normatif lié à la conception des appareils de gammagraphie :

- Norme NF M60-551 (juin 1983) : «Conception des appareils de radiographie gamma ». L'Organisation internationale de normalisation est une fédération mondiale d'organismes nationaux de normalisation dans laquelle la France est représentée par l'Association française de normalisation (AFNOR). La norme internationale relative à la conception du gammagraphe et de ses accessoires repose sur l'ISO 3999 : « appareillage pour radiographie gamma industrielle - spécifications de performance, de conception et d'essais (2004) ». La norme française relative à la conception du gammagraphe et de ses accessoires repose sur la NF M60-551 qui précise :

- Lesdifférences entrenormelaNF etlanormeISOcorrespondante (référence : ISO 3999 de 1977) : limites de débit de dose au contact du projecteur; essais d'étanchéité, cintrage, écrasement, traction pour les gaines d'éjection et accessoires (NF) ; essais d'endurance et résistance à l'effraction pour les verrouillages de l'appareil (NF).

- Le domaine d'application: règles pour la construction des appareils; appareils équipés de sources scellées radioactives (M 61-002).

- Les spécifications qui concernent :

- les limites de débit de dose pour les différentes classes de projecteurs $(\mathrm{P}, \mathrm{M}, \mathrm{F})$;

- la sécurité : verrouillages (manuel du projecteur, automatique du dispositif d'obturation) ; signalisation de la position de la source et de l'obturateur (signal vert : porte-source en position de stockage et obturation complète et verrouillée; signal jaune : porte-source en position de stockage et obturation complète mais non verrouillée ; signal rouge : porte-source en position de stockage ou non, obturation non complète); doublage d'un dispositif de secours manuel de retour de source en position de stockage pour les appareils équipés de télécommandes «non manuelles»; déchargement du porte-source et de la source ;

○ le porte-source ;

- la gaine d'éjection et les accessoires ;

- le dispositif de manutention : projecteurs élingables ;

- la résistance aux conditions de service (projecteur : essais de vibration, de chocs, d'endurance ; télécommande ; gaines d'éjection et accessoires : étanchéité, cintrage, écrasement, traction ; organes de liaison des portes sources) ;

- la résistance aux conditions accidentelles (débit de dose de fuite $<10 \mathrm{mGy} / \mathrm{h}$ à $1 \mathrm{~m})$; 
- le démontage de l'appareil (personne autorisée, outil spécial) ;

- le matériau constituant la protection radiologique (et l'essai d'endurance à la corrosion).

- Les conditions d'essais et les méthodes de mesurage.

- Les indications sur le projecteur et le porte-source(indications gravées sur des plaques résistantes au feu et à l'eau) : sur le projecteur (constructeur, charge max., $\mathrm{n}^{\circ}$ d'identification, activité max. du $\mathrm{RN}$, norme, mention radioactive, mention A - autorisation d'utilisation, mention « ne pas stationner ») ; sur le porte-source (mention « tête de mort » et « radioactive »).

- L'identification de la source (indications gravées sur une plaque résistante au feu et à l'eau) : symbole chimique, activité, date, $\mathrm{n}^{\circ} \mathrm{d}^{\prime}$ identification.

- Les documents d'accompagnement: les descriptions et caractéristiques techniques de l'appareil; le mode d'emploi et de vérification; la maintenance.

Il faut également noter l'aspect normatif lié au balisage et à la signalisation :

- Norme NF M60-101 (décembre 1972) relative à la signalisation des rayonnements ionisants (référence : ISO /R 361 - schéma de base pour les RI). Elle indique le schéma de base normalisé (trisecteur radioactif).

- Norme NF M60-103 (juin 1976) : « Signalisation des rayonnements ionisants, bandes de balisage pour la délimitation de zones particulières pouvant présenter des risques radiologiques ». Elle indique dans ce cadre :

- Les spécifications du balisage : le schéma de base (trisecteur de la norme NF M60-101) et les inscriptions « Franchissement réglementé 》 (couleur jaune, trisecteur noir) ou «Franchissement interdit» (couleur rouge, trisecteur blanc), ainsi que les dimensions des bandes et les couleurs.

- Les conditions d'utilisation : la norme AFNOR M 60-103 définit les bandes de balisage à usage occasionnel (si le risque est permanent, des barrières ou une délimitation matérialisée sont requises hors situation exceptionnelle). Elle prévoit l'utilisation de bandes de balisage de deux couleurs distinctes : jaune pour signaler que le franchissement est réglementé (limitation du séjour du personnel, port d'EPI) et rouge pour signaler que le franchissement est interdit. Elle précise que le séjour dans une zone à franchissement interdit nécessite une autorisation exceptionnelle dûment notifiée dans chaque cas (en pratique, les opérateurs de radiographie gamma sur chantier n'utilisent que des bandes de balisage de couleur rouge).

- Norme NF X08-003 (décembre 1994) : «Couleurs et signaux de sécurité ». La norme NF X 08.003 définit les couleurs et signaux de sécurité, c'est-àdire pour ce qui concerne le signal d'avertissement de l'existence de matières radioactives, le triangle à fond jaune bordé de noir et dont le symbole est le trèfle noir normalisé. 
- Enfin, l'ISO (International Standardisation Organisation) a normalisé un pictogramme destiné à avertir toute personne - en particulier non spécialiste - du danger présenté par une source radioactive. C'est un triangle sur fond rouge contenant le symbole de la radioactivité (le « trèfle ») avec l'indication de l'émission de rayonnements, une tête de mort et un homme qui s'enfuit. Ce pictogramme peut être utilisé en supplément du « trèfle radioactif ».

\section{Principales dispositions réglementaires de radioprotection applicables en gammagraphie}

L'Autorité de sûreté nucléaire (ASN) a rappelé aux industriels, par courrier du 26 avril 2004 (lettre DGSNRSD8/n086/2004, confirmée par la lettre de rappel du 29 décembre 2005), la nécessité de respecter les dispositions règlementaires concernant l'activité de gammagraphie industrielle.

Ce référentiel reprend donc les éléments de l'annexe de la lettre du 26 avril 2004 et les complète en rappelant les principales dispositions règlementaires de radioprotection applicables en gammagraphie balayées au travers des thèmes suivants :

- autorisation et qualification pour la détention et l'utilisation des sources,

- classement des travailleurs et formation,

- surveillance et limites d'exposition du personnel,

- organisation de la radioprotection et de la prévention des risques,

- zonage et signalisation,

- utilisation, stockage, et contrôle des appareils de radiographie industrielle.

\subsection{Autorisation et qualification pour la détention et l'utilisation des sources}

\subsubsection{Demande d'autorisation de détention et d'utilisation de sources}

La détention et l'utilisation d'appareils de radiographie industrielle équipés de sources scellées (ou de générateurs $\mathrm{X}$ pour les cas traités ici) sont soumises à autorisation préalable (articles R.1333-26 et R.1333-27 du Code de la santé publique introduits par le décret 2002-460 du 4 avril 2002, et décret 2006-1454 du 24 novembre 2006 modifiant la nomenclature des installations classées), en considérant pour un même établissement les cas suivants :

- $1^{\mathrm{er}}$ cas : L'établissement est réglementé par un arrêté préfectoral parce qu'il est soumis à autorisation pour une rubrique quelconque de la nomenclature ICPE. Son activité « détention et utilisation de sources radioactives » pour la gammagraphie sera réglementée par cet arrêté sous la rubrique 1715 . Nota : cet arrêté ne réglemente que l'installation contenue dans le périmètre géographique 
de l'établissement. Autrement dit, il n'autorise pas à intervenir sur un chantier extérieur à l'entreprise.

- $2^{\mathrm{e}}$ cas : Aucune installation de l'établissement n'est soumise à autorisation pour une quelconque rubrique de la nomenclature ICPE. Dans ce cas, l'autorisation pour la détention et l'utilisation sur site de sources radioactives est délivrée par l'ASN.

- $3^{\mathrm{e}}$ cas : Les sources sont utilisées sur chantiers extérieurs. L'autorisation est toujours délivrée par l'ASN.

- $4^{\mathrm{e}}$ cas : L'entreprise utilise des générateurs X. L'autorisation est délivrée par l'ASN quel que soit le lieu d'utilisation (établissement ou chantier extérieur).

Pour les établissements réglementés par arrêté préfectoral, le dossier doit être déposé à la préfecture du département et le service instructeur est la DRIRE (Direction régionale de l'industrie, de la recherche et de l'environnement) compétente.

Pour les autorisations délivrées par l'ASN, le dossier doit être déposé à la division compétente de l'ASN, au siège de l'ASN et à l'IRSN (Institut de radioprotection et de sûreté nucléaire). Les formulaires de demande peuvent être téléchargés sur le site www.asn.fr.

L'ouverture d'un chantier de contrôle radiographique de durée prévisible supérieure à un mois doit faire l'objet d'une déclaration auprès de l'inspection du travail, du préfet du département dans lequel le chantier est prévu et de l'autorité administrative ayant délivré l'autorisation prise en application de l'article L.1333-4 du Code de la santé publique. Cette déclaration doit être faite au plus tard 48 heures avant le premier contrôle radiographique (article R.1333-33 du Code de la santé publique et arrêté du 2 mars 2004).

\subsubsection{Qualification de l'entreprise}

Les entreprises qui utilisent des appareils émettant des rayonnements ionisants (comme les appareils de radiographie industrielle) doivent avoir obtenu un certificat de qualification délivré par un organisme accrédité qui justifie de leur capacité à effectuer des travaux sous rayonnements ionisants (article R.231-110 du Code du travail).

Il faut cependant souligner qu'un arrêté conjoint des ministres chargés du travail et de l'agriculture, pris après avis de l'Autorité de sûreté nucléaire et de l'Institut de radioprotection et de sûreté nucléaire, devra déterminer, entre autres, les modalités et conditions d'accréditation des organismes chargés de la certification et la liste des activités ou des catégories d'activité pour lesquelles cette certification est requise en tenant compte de la nature et de l'importance du risque (Article R.231-110. - I et II du Code du travail). 


\subsubsection{Fin d'utilisation de la source}

Tout utilisateur de sources est tenu de faire reprendre celles dont il n'a plus usage ou qui sont périmées (c'est-à-dire 10 ans au plus tard après la date du premier visa apposé sur le formulaire de fourniture), sauf prolongation accordée par l'ASN par délégation du ministre chargé de la santé. Le fournisseur de sources est dans l'obligation de récupérer, sans condition et sur simple demande de l'utilisateur, toute source dont celui-ci n'a plus d'usage ou est périmée (article R.1333-52 du Code la santé publique introduit par le décret 2002-460).

\subsubsection{Déclaration d'incident ou d'accident}

Tout incident ou accident susceptible de porter atteinte à la santé des personnes par exposition aux rayonnements ionisants doit être déclaré sans délai à l'autorité administrative ayant délivré l'autorisation de détenir et d'utiliser des radionucléides (article L.1333-3 du Code de la santé publique).

Pour ce qui concerne les activités nucléaires soumises à un régime d'autorisation ou de déclaration en application de l'article L.1333-4 du Code de la santé publique, le chef d'établissement doit déclarer tout événement significatif ayant entraîné ou étant susceptible d'entraîner le dépassement d'une des limites d'exposition à l'Autorité de sûreté nucléaire (article R.231-105-1 du Code du travail introduit par le décret 2007-1570). Il doit aussi procéder à l'analyse de ces évènements afin d'en prévenir de futurs.

Il faut préciser qu'une décision de l'Autorité de sûreté nucléaire, homologuée par les ministres chargés du travail et de l'agriculture, devra fixer les critères définissant l'événement significatif ainsi que les critères de déclaration et de gestion de ces évènements par le chef d'établissement, compte tenu de la nature et de l'importance du risque.

À ce titre, il est nécessaire de rappeler que l'ASN a proposé à la fin de l'année 2005 un guide (disponible en ligne sur son site) destiné aux exploitants nucléaires concernant les modalités de déclaration des évènements significatifs impliquant la sûreté nucléaire, la radioprotection et les préoccupations relatives à l'environnement applicables aux installations nucléaires de base (INB) ainsi qu'au transport de matières radioactives. L'ASN a également réalisé plus récemment le guide ASN/DEU/03 (version du 15/06/07) relatif aux modalités de déclaration et à la codification des critères relatifs aux évènements significatifs dans le domaine de la radioprotection, hors installations nucléaires de base et transports de matières radioactives, destiné aux responsables d'activités nucléaires telles que définies par l'article L.1333-1 du Code de la santé publique et aux chefs d'établissements dans lesquels sont utilisés des rayonnements ionisants. Il est consultable sur le site http://www.asn.fr/sections/ rubriquesprincipales/publications/publications-pour/listes-publications. 


\subsubsection{Déclaration de perte ou de vol d'une source}

La perte ou le vol d'une source doit être immédiatement déclarée au préfet du département du lieu de survenance de la perte ou du vol (article R.1333-51 du Code la santé publique introduit par le décret 2002-460, art. R.43-46). Le préfet informe l'autorité qui a délivré l'autorisation et l'IRSN.

L'ASN a ouvert une ligne téléphonique spéciale (numéro vert 0800804 135) destinée à recevoir tous les appels signalant des incidents mettant en jeu des sources de rayonnements ionisants utilisées hors INB. Ce numéro vert est accessible 24 heures sur 24, 7 jours sur 7, et les informations fournies lors de l'appel sont transmises à un responsable de l'ASN qui gérera l'incident.

L'IRSN dispose également d'un ingénieur d'astreinte qui assure une permanence 24 h/24 (tél. : +33 (0)6 07315663 ) concernant les situations d'urgence radiologique (comprenant un incident ou accident au cours de l'utilisation d'appareils radiologiques en milieux industriel, une découverte fortuite d'une source radioactive suite à son abandon, sa perte ou son vol, etc.).

\subsection{Classement des travailleurs et formation}

\subsubsection{Classement des travailleurs}

Sont considérés comme personnes du public les personnels non exposés aux rayonnements ionisants, en particulier tous ceux qui ne participent pas à leur mise en œuvre.

Les travailleurs susceptibles de recevoir, dans les conditions habituelles de travail, une dose efficace supérieure à $6 \mathrm{mSv}$ sur 12 mois glissants sont classés par le chef d'établissement en catégorie A, après avis du médecin du travail. En ce qui concerne les travailleurs exposés aux rayonnements ne relevant pas de la catégorie A, ils sont classés en catégorie B (article R.231-88 du Code du travail introduit par le décret 2003-296).

\subsubsection{Formation et information des opérateurs}

Tous les opérateurs susceptibles d'intervenir dans des zones surveillées ou contrôlées doivent bénéficier d'une formation à la radioprotection qui doit être renouvelée au moins tous les trois ans (article R.231-89 du Code du travail introduit par le décret 2003-296). Cette formation doit informer des procédures générales de radioprotection mises en œuvre dans l'établissement (ou les établissements où sont réalisés les chantiers) et des règles de conduite à tenir en cas de situation anormale. Lorsque les travailleurs sont susceptibles d'être exposés à des sources de haute activité telles que mentionnées à l'article R.1333-33 du Code de la santé 
publique (renvoi au tableau C de l'annexe 13-8 du Code de la santé publique), cette formation doit être renforcée, en particulier sur les aspects relatifs à la sûreté et aux conséquences possibles de la perte du contrôle adéquat des sources (article 9 du décret 2007-1570 modifiant l'article R.231-89 du Code du travail). Les utilisateurs de gammagraphes doivent donc être formés aux situations incidentelles et accidentelles probables et informés de leurs conséquences potentielles.

Le chef d'établissement doit également remettre aux opérateurs intervenant en zone contrôlée une notice rappelant les risques particuliers liés au poste de travail qu'ils vont occuper ou à l'intervention qu'ils vont réaliser, les règles de sécurité applicables, ainsi que les instructions à suivre en cas de situation anormale et les coordonnées de la personne compétente en radioprotection (article R.231-90 du Code du travail introduit par le décret 2003-296).

La manipulation d'un appareil de radiographie industrielle ne peut être confiée qu'à des personnes titulaires d'un certificat d'aptitude (CAMARI, Certificat d'aptitude à manipuler les appareils de radioscopie industrielle et radiographie industrielle) valide (article R.231-91 du Code du travail introduit par le décret 2003-296 et arrêté du 25 juin 1987). Il est important de souligner que le terme de « manipulation » a été défini comme «toute action susceptible de modifier les conditions d'exposition des travailleurs aux rayonnements ionisants lors de la mise en œuvre d'un appareil de radiologie » (article 1. de l'arrêté du 21 décembre 2007 définissant les modalités de formation et de délivrance du certificat d'aptitude à manipuler les appareils de radiologie industrielle).

Tout assistant d'un opérateur de gammagraphie doit être en possession d'un CAMARI s'il est amené à manipuler l'appareil. La présence d'un assistant est obligatoire pour tout contrôle radiographique effectué en dehors de l'établissement domiciliaire de l'autorisation de détention et d'utilisation de sources radioactives (arrêté du 2 mars 2004).

Jusqu'au 28 juin 2008, les épreuves du CAMARI sont organisées par les directions régionales du travail et de l'emploi dans les mêmes conditions que précédemment. Au-delà de cette date, c'est l'IRSN qui organisera l'examen du CAMARI, dans les conditions fixées par l'arrêté du 21 décembre 2007 et consultable sur le site http://www.irsn.org/index.php?position=camari.

Pour mémoire, les CAMARI obtenus avant le 28 juin 2008 ont une durée de validité de 9 ans. À partir du second semestre 2008, le CAMARI délivré par l'IRSN aura sa validité maximale ramenée à 5 ans. Dans 1'hypothèse où il existerait des CAMARI ne comportant pas de date de délivrance permettant de déterminer la limite de validité, celle-ci ne pourra pas dépasser 5 ans à partir du 28 juin 2008 . 


\subsubsection{Dispositions règlementaires pour les travailleurs mineurs et les femmes enceintes}

Il est interdit d'occuper les jeunes travailleurs de moins de dix-huit ans aux travaux exposants aux rayonnements ionisants et de les admettre de manière habituelle dans les locaux affectés à ces travaux (article R.234-20 du Code du travail).

Il convient de préciser que les jeunes en apprentissage peuvent être exposés aux rayonnements ionisants (dans les limites de doses relatives à un classement en catégorie B). Ainsi, les personnes âgées de seize à dix-huit ans autorisées lors de leur formation, dans les conditions prévues à l'article R.234-22 du Code du travail, à être occupées à des travaux les exposant aux rayonnements ionisants ne peuvent recevoir au cours de douze mois consécutifs une dose efficace supérieure à $6 \mathrm{mSv}$ ou des doses équivalentes supérieures aux valeurs suivantes :

- $150 \mathrm{mSv}$ pour les mains, les avant-bras, les pieds et les chevilles ;

- $150 \mathrm{mSv}$ pour la peau. Cette limite s'applique à la dose moyenne sur toute surface de $1 \mathrm{~cm}^{2}$, quelle que soit la surface exposée ;

- $50 \mathrm{mSv}$ pour le cristallin (article R.231-77 du Code du travail). Cette valeur est corrigée à $45 \mathrm{mSv}$ pour le cristallin à partir du $1^{\mathrm{er}}$ mai à l'article D.4153-34 du nouveau Code du travail.

En cas de grossesse, l'exposition de la femme enceinte et celle de l'enfant à naître doit être aussi faible que raisonnablement possible et doit être en dessous de $1 \mathrm{mSv}$ pendant le temps qui s'écoule entre la déclaration de la grossesse et le moment de l'accouchement (article R.231-77 du Code du travail introduit par le décret 2003-296).

\subsection{Surveillance et limites d'exposition du personnel}

\subsubsection{Dosimétrie}

Chaque travailleur intervenant en zone surveillée ou contrôlée doit faire l'objet d'un suivi dosimétrique (dosimétrie passive) pour l'estimation de son exposition externe. En outre, s'il intervient en zone contrôlée, il devra bénéficier d'une dosimétrie opérationnelle (articles R.231-93 et R.231-94 du Code du travail insérés par le décret 2003-296). La dosimétrie opérationnelle doit être réalisée à l'aide de dosimètres électroniques permettant d'intégrer puis de lire les doses reçues et comportant des seuils d'alarme.

L'étalonnage des dosimètres opérationnels doit être contrôlé au moins une fois par an (article 2 de l'arrêté du 26 octobre 2005). 


\subsubsection{Suivi médical}

Aucun travailleur ne peut être affecté à un poste exposé s'il n'a pas au préalable bénéficié d'un examen médical permettant au médecin du travail de se prononcer sur son aptitude à occuper ce poste (article R.231-98 du Code du travail introduit par le décret 2003-296).

Les travailleurs classés en catégorie A ou B sont soumis à une surveillance médicale spéciale et bénéficient d'un examen médical au moins une fois par an (article R.231-100 du Code du travail introduit par le décret 2003-296).

Une carte individuelle de suivi médical doit être remise par le médecin du travail à tout travailleur de catégorie $\mathrm{A}$ ou $\mathrm{B}$. Son contenu est fixé par l'arrêté du 30 décembre 2004 (article R.231-102 du Code du travail introduit par le décret 2003-296).

\subsubsection{Limites règlementaires d'exposition aux rayonnements ionisants}

La dose efficace reçue par les travailleurs exposés aux rayonnements ionisants, dont les opérateurs de gammagraphie, ne doit pas dépasser $20 \mathrm{mSv}$ sur 12 mois consécutifs (article R.231-76 du Code du travail inséré par l'article 5 du décret 2003-296).

En outre, une information de l'inspecteur du travail et du médecin du travail doit être également effectuée si les limites règlementaires d'exposition des travailleurs ont été dépassées (article R.231-96 du Code du travail inséré par le décret 2003-296).

La dose efficace annuelle reçue par les personnes du public est limitée à $1 \mathrm{mSv}$ (article R.1333-8 du Code de la santé publique).

Les salariés sous contrat de travail à durée déterminée ou sous contrat temporaire ne peuvent pas être affectés à des travaux sous rayonnements ionisants dès lors que ceux-ci sont effectués dans des zones où le débit de dose horaire est susceptible d'être supérieur à $2 \mathrm{mSv}$ (arrêté du 8 octobre 1990 fixant la liste des travaux pour lesquels il ne peut être fait appel aux salariés sous contrat de travail à durée déterminée ou aux salariés des entreprises de travail temporaire modifié par l'arrêté du 12 mai 1998, et arrêté du 21 juillet 1998 modifiant l'arrêté du 27 juin 1991 modifié).

Lorsqu'un salarié sous contrat à durée déterminée ou sous contrat temporaire est exposé à des rayonnements ionisants et qu'au terme de son contrat cette exposition excède la valeur limite annuelle rapportée à la durée du contrat (prorata temporis), l'employeur est tenu de lui proposer une prorogation (prolongation) 
du contrat (ou un nouveau contrat pour une entreprise de travail temporaire) pour une durée telle que l'exposition constatée à l'expiration de la prorogation soit au plus égale à la valeur limite annuelle rapportée à la durée totale du contrat (articles L.122-3-17 et L.124-22 du Code du travail).

\subsection{Organisation de la radioprotection et de la prévention des risques}

\subsubsection{Optimisation de la radioprotection}

L'exposition des personnes aux rayonnements ionisants doit être maintenue au niveau le plus bas qu'il est raisonnablement possible d'atteindre, en application du principe d'optimisation (articles L.1333-1 du Code de la santé publique et R.231-75 du Code du travail).

\subsubsection{Fiche d'exposition}

Le chef d'établissement établit pour chaque salarié une fiche d'exposition mentionnant la nature du travail à effectuer, les caractéristiques de la source émettrice ; la nature des rayonnements ionisants, les périodes d'exposition ainsi que les autres risques auxquels le salarié est exposé à son poste de travail. Chaque salarié est informé de l'existence de cette fiche et y a accès (article R.231-92 du Code du travail introduit par le décret 2003-296).

\subsubsection{Prévention et coordination de la radioprotection}

Le chef d'établissement doit prendre les mesures nécessaires pour assurer la prévention des accidents du travail et faire appliquer les mesures de prévention nécessaires à la protection de son personnel (article R.231-74-I du Code du travail introduit par le décret 2003-296).

Lorsque le chef d'une entreprise utilisatrice fait intervenir une entreprise extérieure ou un travailleur non salarié, il doit assurer la coordination générale des mesures de prévention qu'il prend et de celles prises par le chef de l'entreprise extérieure ou le travailleur non salarié. À cet effet, le chef de l'entreprise utilisatrice communique à la personne ou au service compétent en radioprotection les informations qui lui sont transmises par les chefs des entreprises extérieures. Il transmet les consignes particulières applicables en matière de radioprotection dans l'établissement aux chefs des entreprises extérieures qui les portent à la connaissance des personnes compétentes en radioprotection qu'ils ont désignées (article R.231-74-II du Code du travail introduit par le décret 2003-296 et modifié par le décret 2007-1570).

Le chef de l'entreprise utilisatrice assure la coordination générale des mesures de prévention et chaque chef d'entreprise est responsable de l'application des 
mesures de prévention nécessaires à la protection de son personnel (article R.237-2 du Code du travail introduit par le décret 92-158).

Dans le cadre de travaux effectués dans un établissement par une entreprise extérieure, il est procédé, préalablement à l'exécution de l'opération, à une inspection commune des lieux de travail et de l'installation (article R.237-6 du Code du travail inséré par le décret 92-158). Lorsque des risques existent, les chefs d'entreprises arrêtent d'un commun accord, avant le début des travaux, le plan de prévention définissant les mesures qui doivent être prises pour chaque entreprise en vue de prévenir ses risques. Les mesures prévues par le plan de prévention comportent au moins des dispositions dans les domaines suivants (article R.237-7 du Code du travail inséré par le décret 92-158) :

- la définition des phases d'activité dangereuses et des moyens de prévention spécifiques correspondants ;

- l'adaptation des matériels, installations et dispositifs à la nature des opérations à effectuer ainsi que la définition de leurs conditions d'entretien ;

- les instructions à donner aux salariés ;

- l'organisation mise en place pour assurer les premiers secours en cas d'urgence et la description du dispositif mis en place à cet effet par l'entreprise utilisatrice ;

- les conditions de participation des salariés d'une entreprise aux travaux réalisés par une autre.

Le chef d'entreprise « extérieure » doit, avant le début des travaux et sur le lieu de leur exécution (article R.237-11 du Code du travail introduit par le décret 92-158) :

- faire connaître à l'ensemble des salariés affectés aux travaux les dangers spécifiques auxquels ils sont exposés et les mesures prises pour les prévenir ;

- préciser les zones dangereuses ainsi que les moyens adoptés pour les matérialiser ;

- expliquer l'emploi des dispositifs collectifs et individuels de protection ;

- montrer les voies d'accès au lieu d'intervention et les issues de secours.

\subsection{4. Évaluation des risques}

Dans le cadre de l'évaluation des risques, l'employeur, en collaboration, le cas échéant, avec le chef de l'entreprise extérieure ou le travailleur non salarié, doit procéder à une analyse des postes de travail qui doit être renouvelée périodiquement et à l'occasion de toute modification des conditions pouvant affecter la santé et la sécurité des travailleurs. Lors d'une opération se déroulant dans la zone 
contrôlée l'employeur (article R.231-75 du Code du travail) :

- fait procéder à une évaluation prévisionnelle de la dose collective et des doses individuelles que les travailleurs sont susceptibles de recevoir lors de l'opération ;

- fait définir par la personne compétente en radioprotection des objectifs de dose collective et individuelle pour l'opération, fixés au niveau le plus bas possible compte tenu de l'état des techniques et de la nature de l'opération à réaliser et, en tout état de cause, à un niveau ne dépassant pas les valeurs limites. À cet effet, les responsables de l'opération doivent apporter leur concours à la personne compétente en radioprotection;

- fait mesurer et analyser les doses de rayonnement effectivement reçues au cours de l'opération. Lorsque la technique le permet, ces mesures sont effectuées de manière continue pour permettre une lecture immédiate de leurs résultats.

\subsubsection{Personne compétente en radioprotection}

Dans tout établissement au sein duquel la présence de source de rayonnements ionisants, telle qu'une source radioactive scellée, est susceptible d'entraîner un risque d'exposition notamment pour ses salariés, le chef d'établissement doit désigner au moins une personne compétente en radioprotection qui devra avoir suivi au préalable et avec succès une formation à la radioprotection (formation de personne compétente en radioprotection). Le chef d'établissement doit mettre à la disposition de la personne compétente en radioprotection les moyens nécessaires à l'exercice de ses missions (article R.231-106-III du Code du travail introduit par le décret 2003-296 et modifié par le décret 2007-1570).

Plus généralement, le chef d'établissement met à disposition de la personne titulaire de l'autorisation les moyens nécessaires pour atteindre et maintenir un niveau optimal de radioprotection du public (article R.1333-7 du Code de la santé publique).

La personne compétente en radioprotection doit procéder à une analyse des postes de travail pour identifier et quantifier le risque encouru par les travailleurs exposés. À partir de cette analyse, elle définit les mesures de protection adaptées à mettre en œuvre et en vérifie leur pertinence, notamment au vu des résultats de la dosimétrie opérationnelle (article R.231-106-1 du Code du travail introduit par le décret 2003-296 et modifié par le décret 2007-1570).

En cas de dépassement d'une limite règlementaire d'exposition aux rayonnements ionisants, la personne compétente en radioprotection, sous la responsabilité du chef d'établissement, doit engager plusieurs actions spécifiques, notamment formaliser l'étude des circonstances dans lesquelles le dépassement s'est produit, ainsi que définir les mesures à prendre pour éviter une nouvelle surexposition (article R.231-97 du Code du travail introduit par le décret 2003-296). 


\subsection{Zonage et signalisation}

\subsubsection{Zonage - Délimitation des zones réglementées}

Le chef d'établissement détenteur d'une source de rayonnement ionisant délimite autour de la source (article R.231-81 du Code du travail introduit par le décret 2003-296) :

- une zone surveillée dès lors que les travailleurs sont susceptibles de recevoir, dans les conditions normales de travail, une dose efficace dépassant $1 \mathrm{mSv}$ par an ;

- une zone contrôlée dès lors que les travailleurs sont susceptibles de recevoir, dans les conditions normales de travail, une dose efficace de $6 \mathrm{mSv}$ par an.

Ces zones sont définies comme suit (articles 5 et 7 de l'arrêté du 15 mai 2006) :

- la zone est désignée surveillée tant que la dose efficace susceptible d'être reçue en une heure reste inférieure à $7,5 \mu \mathrm{Sv}$; au-delà et jusqu'à $25 \mu \mathrm{Sv}$, la zone est désignée zone contrôlée verte ;

- la zone est désignée zone contrôlée jaune tant que la dose efficace susceptible d'être reçue en une heure reste inférieure à $2 \mathrm{mSv}$ et que le débit d'équivalent de dose ne dépasse pas $2 \mathrm{mSv} / \mathrm{h}$ pour l'exposition externe du corps entier;

- la zone est désignée zone contrôlée orange tant que la dose efficace susceptible d'être reçue reste inférieure à $100 \mathrm{mSv}$ et que le débit d'équivalent de dose ne dépasse pas $100 \mathrm{mSv} / \mathrm{h}$ pour l'exposition externe du corps entier ;

- la zone est désignée zone interdite rouge lorsque la dose efficace susceptible d'être reçues en une heure ou le débit d'équivalent de dose sont égaux ou supérieurs à l'une des valeurs maximales définies pour la zone orange.

À ces conditions de délimitations de zones s'ajoutent des limites de doses équivalentes susceptibles d'être reçues en une heure aux extrémités (articles 5 et 7 de l'arrêté du 15 mai 2006).

Lorsque l'émission de rayonnements ionisants n'est pas continue, comme pour la radiographie industrielle à poste fixe ou en « bunker », la délimitation de la zone contrôlée peut être intermittente mais la zone considérée doit être a minima une zone surveillée quand l'émission de rayonnements ionisants ne peut être exclue (c'est-à-dire quand l'appareil n'est pas « verrouillé »). Si l'appareil est verrouillé sur une position interdisant toute émission de rayonnements ionisants, le zonage peut être suspendu temporairement (article 9 de l'arrêté du 15 mai 2006).

Lorsqu'une opération, notamment de maintenance, est susceptible de modifier l'intégrité des protections autour de la source, le chef d'établissement doit faire évoluer le zonage en fonction des nouvelles conditions d'exposition aux rayonnements ionisants et la zone nouvellement délimitée est a minima une zone surveillée le temps de l'intervention (article 10 de l'arrêté du 15 mai 2006). 
Dans le cas de l'utilisation d'un appareil mobile ou portable (cas de l'utilisation de gammagraphes sur chantiers « extérieurs »), le responsable de l'appareil doit délimiter une zone d'opération (l'article 13 de l'arrêté du 15 mai 2006 précise que cette zone est une zone contrôlée) autour de l'appareil de gammagraphie telle que, à la périphérie de celle-ci, le débit d'équivalent de dose moyen, évalué sur la durée d'opération, reste inférieur à $2,5 \mu \mathrm{Sv} / \mathrm{h}$. La notion d'opération n'est pas définie règlementairement, mais une circulaire apporte une interprétation de ce terme. À titre exceptionnel et lorsque les conditions techniques de l'opération rendent impossible la mise en place des dispositifs de protection radiologique, ou si ces dispositifs n'apportent pas une atténuation suffisante, le débit d'équivalent de dose moyen, évalué sur la durée de l'opération, peut être supérieur à la limite citée précédemment sans jamais dépasser $25 \mu \mathrm{Sv} / \mathrm{h}$. Dans ce cas, le responsable de l'appareil établit préalablement à l'opération, le cas échéant en concertation avec le chef de l'entreprise utilisatrice, un protocole spécifique (dont le contenu est précisé par l'arrêté du 15 mai 2006) qui devra être porté à la connaissance de tous les travailleurs en charge de l'opération (articles 13 et 14 de l'arrêté du 15 mai 2006).

Lorsque l'appareil est mis en œuvre à l'intérieur d'une zone surveillée ou contrôlée, la délimitation de la zone d'opération prend en compte les débits de doses inhérents à l'appareil ainsi que ceux déjà existants dans ces zones. La délimitation de la zone d'opération est alors établie suivant les valeurs d'exposition aux rayonnements ionisants fixées par les articles 5 et 7 de l'arrêté du 15 mai 2006 pour définir les zones contrôlées et surveillées (article 13 de l'arrêté du 15 mai 2006).

\subsubsection{Signalisation}

La zone d'opération doit être délimitée de manière visible et continue tant que l'appareil est en place. Cette zone d'opération doit être signalée par des panneaux installés de manière visible et qui mentionnent la nature du risque et l'interdiction d'accès à toute personne non autorisée. Les panneaux conformes à la norme NF M 60-101 (trisecteur radioactif) sont réputés satisfaire aux prescriptions de l'arrêté du 15 mai 2006. Une signalisation doit avertir le personnel du début et de la fin de l'exposition aux rayonnements ionisants (article 6 de l'arrêté du 2 mars 2004). Pour cela, un dispositif lumineux doit être activé durant la période d'émission des rayonnements ionisants ; il peut être complété, en cas de besoin, par un dispositif sonore. Cette signalisation doit être enlevée en fin d'opération, lorsque l'appareil est verrouillé sur une position interdisant toute émission de rayonnements ionisants (article 16 de l'arrêté du 15 mai 2006).

À l'intérieur d'une zone contrôlée ou surveillée, les sources de rayonnements doivent être signalées (articles 8 de l'arrêté du 15 mai 2006 et R.231-82 du Code du travail introduit par le décret 2003-296). 
Sur un gammagraphe, la source doit être identifiée par une plaquette qui porte les inscriptions suivantes : le symbole chimique et le nombre de masse du radioélément, l'activité du radioélément et la date de sa mesure, ainsi que le numéro d'immatriculation de la source. De plus, le porte source doit porter sur la surface extérieure la mention « Radioactivité » et le symbole « Tête de mort » (articles 17 et 18 du décret 85-968).

\subsection{Utilisation, stockage, et contrôle des appareils de radiographie industrielle}

\subsubsection{Maintenance et contrôle des appareils de radiographie industrielle}

Un carnet d'entretien ou de suivi doit être attribué à chaque appareil de radiographie " gamma » et une fiche de suivi à chaque accessoire. Il est prescrit règlementairement que ces documents doivent accompagner les appareils et être régulièrement tenus à jour, au moins une fois par semaine. Le carnet de suivi devra notamment mentionner toutes les révisions périodiques, les chargements de sources mais aussi les paramètres d'exploitation et les incidents survenus en indiquant leur nature, leurs dates, leurs conséquences (arrêté du 11 octobre 1985).

Les canaux des projecteurs, les gaines d'éjection, les télécommandes et les dispositifs d'irradiation des appareils de radiographie « gamma » doivent être protégés contre la pénétration de tout corps étranger, notamment l'eau et la poussière (article 7 du décret 85-968 du 27 août 1985).

Le chef d'établissement établit le programme des contrôles externes (à réaliser par l'IRSN ou un organisme agrée) et internes (à réaliser par le service compétent en radioprotection de l'entreprise) et le consigne dans un document interne à disposition des agents de contrôle compétents et du CHSCT, ou à défaut les délégués du personnel (article 2 de l'arrêté du 26 octobre 2005).

Le chef d'établissement procède ou fait procéder à un contrôle technique de radioprotection des sources et des appareils émetteurs de rayonnements ionisants, des dispositifs de protection et d'alarme ainsi que des instruments de mesure utilisés. Un contrôle périodique des sources et des appareils émetteurs de rayonnements ionisants doit être effectué au moins une fois par an par l'IRSN ou par un organisme agrée (article R.231-84 du Code du travail inséré par le décret 2003-296).

La périodicité des contrôles techniques internes pour les appareils contenant des sources scellées, comme les appareils de radiographie industrielle, est (article 2 de l'arrêté du 26 octobre 2005) :

- trimestrielle pour les sources de haute activité (à partir de $4 \mathrm{GBq}$ pour le Co-60 et $10 \mathrm{GBq}$ pour l'Ir-192 - seuils définis par la directive euratom 2003/122) ; 
- semestrielle pour les sources scellées dont la classification ne répond pas à celle recommandée par la norme ISO 2919 pour l'utilisation considérée ou pour les sources scellées bénéficiant d'une prolongation d'utilisation au-delà des 10 ans ;

- annuelle pour les sources scellées dont la classification répond à celle recommandée par la norme ISO 2919 pour l'utilisation considérée.

Les instruments de mesure («radiamètres ») et les dispositifs de protection et d'alarme doivent également faire l'objet d'un contrôle interne périodique au moins une fois par an ou avant leur utilisation si ceux-ci n'ont pas été employés depuis plus d'un mois. De même, ils doivent faire l'objet d'un contrôle périodique de l'étalonnage (article 2 de l'arrêté du 26 octobre 2005 et article R.43-3 du Code du travail).

Les résultats de ces contrôles, internes et externes, doivent être consignés dans le document prévu à l'article R.230-1 du Code du travail, qui doit également inclure un relevé des sources et appareils émettant des rayonnements ionisants utilisés ou stockés dans l'établissement. Une copie de ce relevé doit être transmise, au moins une fois par an, à l'IRSN (article 231-87 du Code du travail inséré par le décret 2003-296).

Les contrôles externes et internes font l'objet de rapports écrits, mentionnant la date, la nature et la localisation des contrôles, les noms et qualités de la ou des personnes les ayant effectués ainsi que les éventuelles non-conformités relevées. Ces rapports devront être conservés par le titulaire de l'autorisation pendant une durée de dix ans (article 3 de l'arrêté du 26 octobre 2005).

Les appareils de radiographie portatifs ou mobiles et leurs accessoires doivent faire l'objet au moins une fois par an d'une révision complète, exécutée par des personnels qualifiés, sous la responsabilité de leur constructeur. Les autres appareils doivent subir cette révision lors de chaque rechargement (article 21 du décret 85-968 du 27 août 1988).

\subsubsection{Utilisation des appareils de radiographie industrielle}

Le local ou le chantier où ont lieu les opérations de radiographie doit être débarrassé des objets inutiles susceptibles de diffuser le rayonnement (article 6 de l'arrêté du 2 mars 2004).

L'accès au local ou au chantier doit être matériellement interdit pendant la durée de l'exposition par la mise en place dispositifs ne pouvant être franchis par inadvertance. En cas d'utilisation d'appareils mobiles, la zone où les personnes étrangères à l'opération ne peuvent avoir accès doit être matérialisée (article 6 de l'arrêté du 2 mars 2004).

La position de la source au moment de l'armement et le retour de celle-ci en position de protection doivent être vérifiés lors de chaque opération au moyen d'un détecteur 
de rayonnements (« radiamètre »). Après chaque utilisation, la clé de sécurité doit être retirée sans délai à l'issue de la vérification du retour de la source et être conservée séparée de l'appareil de radiographie (article 6 de l'arrêté du 2 mars 2004).

Un appareil de radiographie ne doit être déplacé - y compris à l'intérieur des limites d'un chantier - que s'il est verrouillé et que si la clé de sécurité est séparée de l'appareil (article 7 de l'arrêté du 2 mars 2004).

\subsubsection{Stockage des appareils de radiographie industrielle}

Les appareils de radiographie mobiles ou portatifs ne doivent pas être laissés sans surveillance adaptée (article 8 de l'arrêté du 2 mars 2004).

Le stockage des appareils de radiographie doit se faire dans un local fermé à clé, à accès contrôlé et les dispositions nécessaires doivent être prises pour assurer la protection contre le vol et l'incendie. Ceci impose qu'un appareil ne peut être stocké dans un véhicule même fermé.

\section{Conclusion}

La gammagraphie est une technique de contrôle non destructif (CND) qui permet d'apprécier les défauts d'homogénéité dans le métal (en particulier dans les cordons de soudure) mais qui présente des risques du fait de l'utilisation de sources radioactives (principalement de l'iridium-192) dont les activités sont élevées (jusqu'à 4,4 TBq en Ir-192 par exemple).

Des incidents (ou simples écarts suivant la définition qu'on donne aux évènements répertoriés), avec ou sans exposition, ont eu lieu en France mais rares sont ceux qui ont entraîné un dépassement des limites règlementaires d'exposition. On peut cependant souligner que ces limites règlementaires d'exposition peuvent être dépassées en quelques minutes en cas d'accident. Dans les cas les plus graves, une surexposition localisée ou même globale peut entraîner l'apparition d'effets déterministes (cas de l'accident de Dakar et Abidjan entre juin et août 2006 : une source est restée bloquée en position intermédiaire dans la gaine sans que les opérateurs ne s'en aperçoivent ; cette situation a entraîné une surexposition de plusieurs personnes, dont certaines gravement atteintes).

Bon nombre d'évènements significatifs de radioprotection liés aux chantiers de gammagraphie impliquent un défaut ou un franchissement (volontaire ou non) de balisage. Malgré les moyens de protection mis en œuvre sur les chantiers, l'organisation et le facteur humain (absence de balisage, non respect des procédures, etc.) interviennent dans une majorité des incidents. Les conditions de travail interviennent également dans l'augmentation du risque d'incident. Les chantiers de tirs 
gammagraphiques sont souvent réalisés dans des conditions difficiles quelque soit le domaine d'activité (bruit environnant, travail nocturne, accessibilité difficile, éclairage, débit de dose, contamination, autres chantiers en parallèle, risque d'incendie ou d'explosion élevé sur certains chantiers, etc.). Enfin, les incidents liés à la perte ou au vol de gammagraphes soulignent également l'importance de la surveillance à associer à la possession d'un gammagraphe. Dans son rapport « Priorités en radioprotection - Propositions pour une meilleure protection des personnes contre les dangers des rayonnements ionisants » remis le 2 mars 2004 à l'ASN, la commission VROUSOS a d'ailleurs identifié dans les « actions à réaliser immédiatement ou à engager sans attendre » le fait de renforcer la qualité et le contrôle de la radioprotection des sources mobiles de haute activité, en particulier dans le domaine de la gammagraphie industrielle, précisant que depuis le 11 septembre 2001, il ne serait pas responsable de sous-estimer le risque de malveillance.

En tout état de cause, la vigilance vis-à-vis de l'activité de gammagraphie reste de mise, au vu, notamment, de l'accident d'irradiation récent d'un travailleur au sein de l'établissement ONERA de Toulouse. Le 12 mars 2008, l'ONERA a constaté un dysfonctionnement du dispositif de protection d'une source radioactive, se trouvant au laboratoire MEGA de son centre de Toulouse. Lors d'une opération de maintenance sur l'irradiateur (de type GMA2500), l'oubli de remise en place de l'embout de la canne d'éjection de la source a conduit à l'expulsion de celle-ci de son coffrage. Cet incident n'a pu être immédiatement détecté, en raison d'une anomalie des systèmes de sécurité. De ce fait, un technicien de la société HIREX, qui effectuait une expérimentation dans ce laboratoire, a été exposé pendant quelques minutes par la source de cobalt-60 tombée au sol. Concernant cet accident, l'ASN précise que l'irradiateur contenant cette source avait été installé et utilisé dans une configuration plusieurs fois modifiée, sans que les dispositifs de sécurité n'aient été vérifiés de manière satisfaisante. Par ailleurs, il a été mis en évidence de nombreux écarts règlementaires en matière de radioprotection, ainsi que des lacunes dans la formation et l'encadrement du personnel en charge des opérations d'irradiation. Compte tenu de ces lacunes, l'ONERA n'a pas été en mesure d'éviter et de déceler immédiatement la perte du contrôle de la source. Des investigations complémentaires sont dès à présent engagées par l'ASN et l'IRSN pour déterminer les circonstances exactes de cet accident, notamment les dysfonctionnements techniques à son origine.

Au vu des évènements récents, il apparaît donc d'autant plus important de rappeler que le respect des prescriptions règlementaires, le renforcement de l'information et de la formation aux risques spécifiques de l'activité de gammagraphie industrielle et enfin les contrôles lors de la réalisation des chantiers de gammagraphie restent des éléments majeurs de la prévention des accidents. 